\title{
Allgemeiner Teil
}

1. Einfahrung . . . . . . . . . . . . . . . . . . . . . . 7

2. Zum Begriff der Bilanz und Ernahhrungebilanz . . . . . . . . . . . . . . . . . . . . . 8

3. Aufbau, Inhalt und Hauptergebnis ernährungsphysiologischer Bilanxnormen . . . . . . . . 10

4. Erfaseung der normativen Lebensmittelkosten im Rahmen von Bilanzen . . . . . . . . . . 16

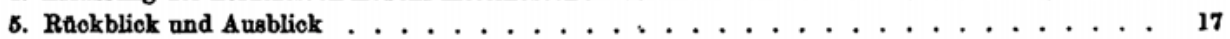

\section{Besonderer Teil}

I. Allgemeines . . . . . . . . . . . . . . . . . . . . . . . . . 21

II. Begründung der zugrundegelegten Ernährungs-Soll- und der ermittelten Ernährungs-IstWerte im einzelnen . . . . . . . . . . . . . . . . . . . . . . . . . . . . 22

A. Hinsichtlioh der Hauptnährstoffe . . . . . . . . . . . . . . . . . . . . . 22

1. Eiweiß .......................... 22

s) Sollsătze . . . . . . . . . . . . . . . . . . . . 22

b) Istabtze . . . . . . . . . . . . . . . . . . . . . . 31

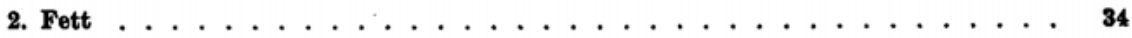

s) Solleătze ............................ 34

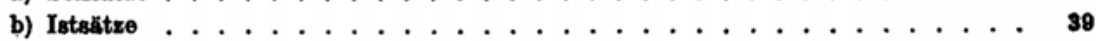

3. Kohlenhydrate ..........................41

B. Hinsiahtlioh der auggewählten Mineral- und Spurenstoffe . . . . . . . . . . . . 41

a) Solleätze ............................... . 41

a) Vorbemerkung . . . . . . . . . . . . . . . . . . . . . 41

8) Calcium . . . . . . . . . . . . . . . . . . . . . . . 44

r) Phosphor ............................ 46

ठ) Eisen . . . . . . . . . . . . . . . . . . . . . . . . . . . 48

b) Istabtze .............................. . . . 40

a) Vorbemerkung . . . . . . . . . . . . . . . . . . 49

в) Caloinm . . . . . . . . . . . . . . . . . . . . . 49

r) Phosphor und Eisen ..................... 51

C. Hinsichtlich der erfaßten Vitamine . . . . . . . . . . . . . . . . . 52

a) Sollsătze . . . . . . . . . . . . . . . . . . . . . . 52

a) Vorbemerkung ........................ 52

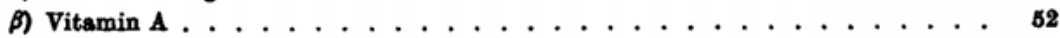

y) Vitamin $B_{1} \ldots \ldots \ldots 6$

8) $\operatorname{Vitsmin} \mathrm{B}_{\mathbf{2}} \ldots \ldots \ldots \ldots$

в) Nicotinsäuresmid . . . . . . . . . . . . . . . . . . . 57

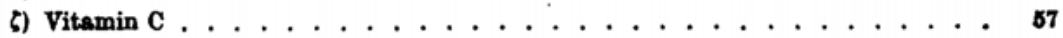


b) Isteätze . . . . . . . . . . . . . . . . . . . . . . 58

a) Vorbemerkung ....................... 58

ק) Vitamin A . . . . . . . . . . . . . . . . . 58

y) Vitamin $B_{1} \ldots \ldots \ldots 1$

ठ) Vitamin $\mathrm{B}_{2} \ldots \ldots \ldots$. . . . . . . . . . . . . . . . . . 62

в) Niootinsäureamid ....................... 62

Ђ) $\operatorname{vitamin~C~........................~} 63$

III. Anhänge . . . . . . . . . . . . . . . . . . . . . . . . . . . 64

A. Einige Bemerkungen zu den typisohen Jahresdurchschnitts-Mittagsmshlzeiten . . . . . . 64

B. Prozentuale Anteile an den Hauptnähratoffen EiweiB, Fett, Kohlenhydrate im AngchluB an die errechneten 13 Ernăhrungsbilanzen . . . . . . . . . . . . . . . 66

\section{Praktisoher Teil}

I. Ernâhrungsbilantnormen in Form von Zahlenabensichten . . . . . . . . . . . . . 67

A. Ernảhrungsbilans fur einen Arbeiter auf der Basis von 3000 Kalorien Tagesbedarf . . 68

B. Emährungsbilans for Mittelsohwerarbeitende anf der Basis von 3400 Kalorien Tagesbedart ..........................

C. Ernährungsbilans for einen Sohwerarbeiter anf der Basis von 3900 Kalorien Tagesbedarf

D. Ernährungsbilanz far einen Sohwerstarbeiter auf der Bagis von 4200 Kalorien Tagesbedart .............................

E. Ernährungsbedarf far einen Schwesstarbeiter auf der Basis von 4500 Kalorien Tagesbedarf

F. Ernährungsbilanz for einen Sohwerstarbeiter auf der Basis von 5000 Kalorien Tagesbedarf

G. Ernährungsbilanz für eine weiblicheBârokraft auf der Basis von 2200 Kalorien Tagesbedarf

H. Ernährungsbilanz fur eine weibliche Bürokraft mit zusätzlich zweiatûndiger Hausarheit auf der Basis von 2400 Kalorien Tagesbedarf . . . . . . . . . . . . . . . . . 82

J. Ernăhrungsbilanz für eine Hausfrau auf der Basis von 2800 Kalorien Tagesbedarf . . . 84

K. Ernährungsbilanz fûr qualifizierte Arbeitskräfte ohne wesentliche physisohe Anstrengung (Facharbeiter) auf der Basis von 2800 Kalorien Tagesbedarf . . . . . . . . 86

L. Ernälurungsbilanz für Kinder von 0 bis 5 Jahren auf der Basis von 1350 Kalorien Tagesbedarf

M. Ernährungsbilanz fưr Kinder von 5 bis 9 Jahren auf der Basis von 1800 Kalorien Tagesbedarf

N. Ernährungsbilanz für Kinder von 9 bis 15 Jahren auf der Basis von 2600 Kalorien Tagesbedarf 70

II. Die gleichen Ernährungsbilanxnormen in graphischer Darstellung

A. Arbeiter: $\mathbf{3 0 0 0}$ Kalorien Tageebedarf

B. Mittelsohwerarbeitende: 3400 Kalorien Tagesbedarf

C. Schwerarbeiter : $\mathbf{3 9 0 0}$ Kalorien Tagesbedarf

D. Schweratarbeiter: 4200 Kalorien Tagesbedarf

E. Schwerstarbeiter: $\mathbf{4 5 0 0}$ Kalorien Tagesbedarf

F. Sohwerstarbeiter: 5000 Kalorien Tagesbedarf

G. Weibliche Bûrokraft: 2200 Kalorien Tageebedarf

H. Weibliche Bürokraft mit zusätzlich zweistondiger Hsusarbeit : 2400 Kalorien Tagesbedarf

J. Hausfrau: 2800 Kalorien Tagesbedarf

K. Qualifizierte Arbeitskräfte ohne wesentliche physische Anstrengung (Faoharbeiter): 2800 Kalorien Tagesbedarf

L. Kinder von 0 bis 5 Jahren: 1350 Kalorien Tagesbedarf

M. Kinder von 5 bis 9 Jahren: 1800 Kalorien Tagesbedarf

N. Kinder von 9 bis 15 Jahren: 2600 Kalorien Tagesbedarf

III. Prozentuale Anteile an den Hauptnährstoffen Eiweiß, Fett, Kohlenhydrate im Ansohluß an die erreohneten 13 Ernährungsbilanzen (in graphisoher Darstellung) 